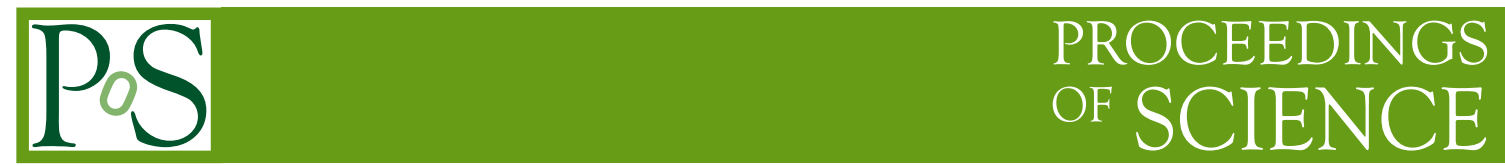

\title{
Quantum measurements and chiral magnetic effect
}

\author{
Vladimir Shevchenko* \\ National Research Centre "Kurchatov Institute" \\ E-mail: Vladimir.Shevchenkodcern.ch
}

The effect of anisotropy for fluctuations of electric currents in magnetic field is addressed within framework of quantum measurements theory. It is shown that for free fermions in uniform magnetic field the anisotropy is of the same sign as one expects for chiral magnetic effect and is related to triangle anomaly. The corresponding decoherence functional contains anomalous off-diagonal terms leading to correlation of fluctuations between observables of opposite P-parity.

Xth Quark Confinement and the Hadron Spectrum,

October 8-12, 2012

TUM Campus Garching, Munich, Germany

\footnotetext{
* Speaker.
} 


\section{Introduction}

One of important questions in quantum field theory is about the fate of discrete symmetries under this or that choice of external conditions. Can one get nontrivial symmetry breaking effect by, for example, heating quantum fields or applying external classical background? Naively all three main discreet symmetries: charge conjugation $\mathbf{C}$, parity reversion $\mathbf{P}$ and time reversion $\mathbf{T}$ are not seen in our everyday experience, as manifested by matter over antimatter dominance in the Universe, bio-chirality, arrow of time and numerous other facts. However as is well known the microscopic situation is much more subtle. Namely, the interactions governing the macro-world long ranged gravity and electromagnetism - are invariant under $\mathbf{C}, \mathbf{P}$ and $\mathbf{T}$, while the microscopic dynamics respect only the famous CPT product: direct $\mathbf{P}$-violation is built into electroweak sector of the Standard Model (lefts are doublets and rights are singlets), while conjugate parity $\mathbf{C P}$ (and hence $\mathbf{T}$ - invariance) is broken by Cabibbo-Kobayashi-Maskawa mechanism.

Strong interactions stay apart in some sense. Leaving aside strong $\mathbf{C P}$-problem and all related issues, QCD Lagrangian without $\theta$-term is invariant under separate $\mathbf{C}$-, $\mathbf{P}$ - and $\mathbf{T}$-transformations. $\mathbf{C}$-invariance holds at finite temperature but gets broken at finite density: there is no Furry theorem if some levels in the upper Dirac continuum are occupied. Strong and quite general results [U, [] guarantee that vacuum expectation value of any local $\mathbf{P}$-nonconserving observable has to vanish in vector-like theories such as QCD, e.g.

$$
\left\langle\bar{\psi} \gamma^{5} \psi\right\rangle=0 ;\left\langle\operatorname{Tr} G_{\mu v} \tilde{G}^{\mu v}\right\rangle=0
$$

Despite these results put serious constraints on possible $\mathbf{P}$-parity violating phenomena in the do-

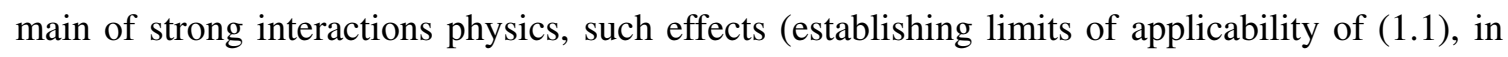
some sense) have been studied for a long time. One can mention T.D.Lee's idea of $\mathbf{P}$-odd bubbles and A.B.Migdal's hypothesis of pion condensate in nuclei. Closely related effects of $\rho-\pi$ mixing at finite temperature [园] and sphaleron dynamics in QCD [䧃] were discussed.

Recently the topic has been revitalized in a series of papers by D.Kharzeev and coauthors [ $[$,

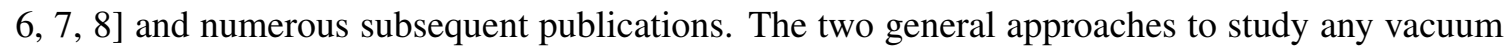
nicely work together in heavy ion collision experiments with respect to the QCD vacuum. Indeed, test particles used in these experiments - heavy ions - are able to create, in the first instants after the collision, highly nontrivial multi-particle state, which itself play a role of external conditions put on the QCD vacuum. These include temperature, density and also extremely strong magnetic field, of the order of $\left(10^{3}-10^{4}\right) \mathrm{MeV}^{2}$ in about $0.2 \mathrm{Fm} / c$ after the moment of collision. This is the first (and perhaps the only) case in physics where one can study strong and electromagnetic interactions interplay on the same scale, without treating the latter as a weak perturbation. The main qualitative result can be formulated as follows: if by whatever dynamical mechanism there is an excess of quarks of definite chirality inside a fireball, it transforms into electric current flowing along the magnetic field, whose main effect is charge asymmetry of final particles distribution between upper and lower (with respect to the interaction plane) hemispheres. On quantitative level, for free massless spinors with charge $e$, chemical potentials $\mu_{L}, \mu_{R}$ for left-handed and right-handed ones, respectively, in constant and spatially uniform magnetic field $\mathbf{B}$ electric current is given by 
the following expression, known as chiral magnetic effect (CME):

$$
\mathbf{j}=\frac{e^{2}}{2 \pi^{2}} \mu_{5} \mathbf{B} ; \mu_{5}=\frac{\mu_{R}-\mu_{L}}{2}
$$

The expression ([L]) first explicitly obtained in [Q] (not in heavy ion collision context) is a robust theoretical result and can be reproduced in many ways. What is special and important about the result $(\mathbb{L 2} 2)$ is that proportionality coefficient there is universal and fixed by the famous triangle anomaly [ய], प]].

In this talk complementary view of the problem is presented using methods of quantum theory of measurements. Throughout the text $\hbar=c=1$, the Minkowski metric tensor is $g_{\mu \nu}=$ $\operatorname{diag}(1,-1,-1,-1)$ and constant uniform magnetic field is chosen in the third direction: $F_{12}=B$. Also the notation $p^{\|}=\left(p^{0}, 0,0, p^{3}\right), p^{\perp}=\left(0, p^{1}, p^{2}, 0\right), p^{\|} \gamma^{\|}=p^{0} \gamma^{0}-p^{3} \gamma^{3}, p^{\perp} \gamma^{\perp}=p^{1} \gamma^{1}+p^{2} \gamma^{2}$ is used for four-vector components parallel and perpendicular to the field and their products.

\section{Current fluctuation asymmetry and quantum measurements}

The simplest case of free massless fermions in external magnetic field at finite temperature is addressed in this section. We are interested to study how their fluctuations (both thermal and quantum) are affected by external field. In principle, this information is encoded in polarization operator $\Pi_{\mu v}(x, y)=i\left\langle T\left\{j_{\mu}(x) j_{v}(y)\right\}\right\rangle$ and we refer an interested reader to [미 for discussion which structures of $\Pi_{\mu v}(x, y)$ in magnetic field correspond to chiral magnetic effect. In what follows we take a different attitude and consider instead the standard Unruh-DeWitt detector coupled to the current. The corresponding Hamiltonian reads:

$$
H=\int_{\tau_{0}}^{\tau_{1}} d \tau \mu(\tau) n^{\mu} \bar{\psi}(x(\tau)) \gamma_{\mu} \psi(x(\tau))
$$

Here $x(\tau)$ parameterizes the detector's world-line, $\tau$ - proper time along it, $n^{\mu}$ - constant vector, fixing a direction the current is measured in, and $\mu(\tau)$ - internal quantum variable of the detector whose evolution in $\tau$ is described by the standard two-level Hamiltonian with the levels $E_{0}$ and $E_{1}$, $E_{1}-E_{0}=\omega>0$. An amplitude for the detector to "click" is given by

$$
\mathscr{A}=i \int_{\tau_{0}}^{\tau_{1}} d \tau\langle 1|\mu(\tau)| 0\rangle \cdot\left\langle\Omega\left|n^{\mu} j_{\mu}(x(\tau))\right| \Omega_{0}\right\rangle
$$

where $j_{\mu}(x(\tau))=\bar{\psi}(x(\tau)) \gamma_{\mu} \psi(x(\tau))$ and $\left|\Omega_{0}\right\rangle$ stays for initial (thermal vacuum) state of the field sub-system, while $|\Omega\rangle$ represents final (after the measurement) state. The corresponding response function reads:

$$
\mathscr{F}(\omega) \equiv n^{\mu} n^{v} \mathscr{F}_{\mu \nu}(\omega)=n^{\mu} n^{v} \int_{\tau_{0}}^{\tau_{1}} d \tau \int_{\tau_{0}}^{\tau_{1}} d \tau^{\prime} e^{-i \omega\left(\tau-\tau^{\prime}\right)} \cdot G_{\mu \nu}^{+}\left(\tau-\tau^{\prime}\right)
$$

where

$$
G_{\mu v}^{+}\left(\tau-\tau^{\prime}\right)=\left\langle\Omega_{0}\left|j_{\mu}(x(\tau)) j_{v}\left(x\left(\tau^{\prime}\right)\right)\right| \Omega_{0}\right\rangle
$$


Usually one is interested in detector excitation rate in unit time. For infinite observation time range $\left(\tau_{0} \rightarrow-\infty, \tau_{1} \rightarrow \infty\right)$ it is determined by the power spectrum of the corresponding Wightman function:

$$
\dot{\mathscr{F}}(\omega)=\int_{-\infty}^{\infty} d s e^{-i \omega s} G^{+}(s)
$$

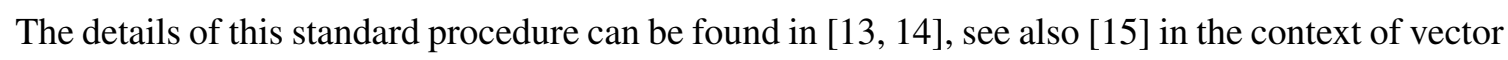
current measurements.

To compute (2.5]) it is convenient to use the exact fermion propagator in external magnetic field given by [ए]]

$$
S(x, y)=e^{i \phi(x, y)} \int \frac{d^{4} p}{(2 \pi)^{4}} e^{i p(x-y)} \tilde{S}(p)
$$

where the gauge-dependent phase $\phi(x, y)$ is irrelevant for gauge-invariant quantities and Fourier transfrom $\tilde{S}(p)$ reads:

$$
\tilde{S}(p)=\int_{0}^{\infty} d u e^{i u\left(p_{0}^{2}-p_{3}^{2}-p_{\perp}^{2} \frac{\tan (q B u)}{q B u}-m^{2}\right)}\left[P_{0}\left(p^{\|}, p^{\perp}\right)+P_{1}\left(p^{\|}\right)\right]
$$

where

$$
P_{0}\left(p^{\|}, p^{\perp}\right)=p^{\|} \gamma^{\|}-p^{\perp} \gamma^{\perp}\left(1+\tan ^{2}(q B u)\right)+m ; P_{1}\left(p^{\|}\right)=\left(p^{\|} \gamma^{\|}+m\right) \gamma^{1} \gamma^{2} \tan (q B u)
$$

and $q$ stays for quark electric charge. We take in the rest of the paper $m=0$. It will be seen that only the tensor structure $P_{1}\left(p^{\|}\right)$is responsible for charge fluctuations asymmetry and also for anomalous $\left\langle j_{\mu} j_{v}^{5}\right\rangle$ correlation.

It is customary in quantum measurements theory to compare response functions of a given detector in a state of inertial movement versus some non-inertial one. We are interested in another kind of asymmetry, namely between the detector oriented to measure current along the magnetic field direction and perpendicular to it. This choice is fixed by the vector $n_{\mu}=(0, \mathbf{n})$. With respect to its spatial movement the detector is supposed to be always at rest, so we can take $x(\tau)=(\tau, 0,0,0)$. Therefore it is convenient to switch to the coordinate space (as in ([2.5]) we denote $s=\tau-\tau^{\prime}$ ):

$$
S(s)=\frac{i s \gamma^{0}}{32 \pi^{2}} \int_{0}^{\infty} \frac{d u}{u^{3}}\left(\frac{q B u}{\tan (q B u)}+\gamma^{1} \gamma^{2} q B u\right) e^{-i \frac{s^{2}}{4 u}}
$$

The response function asymmetry given by $\delta \dot{\mathscr{F}}(\omega)=\dot{\mathscr{F}}_{33}(\omega)-\left(\dot{\mathscr{F}}_{11}(\omega)+\dot{\mathscr{F}}_{22}(\omega)\right) / 2$ is quadratic in $B$ for all values of the magnetic field. ${ }^{1}$ Explicitly, one gets:

$$
\begin{aligned}
& G_{33}^{+}(s)=-\left[\frac{s}{16 \pi^{2}} \int_{0}^{\infty} \frac{d u}{u^{3}} \frac{q B u}{\tan (q B u)} e^{-i \frac{s^{2}}{4 u}}\right]^{2}-\frac{(q B)^{2}}{16 \pi^{4} s^{2}} \\
& G_{11}^{+}(s)=-\left[\frac{s}{16 \pi^{2}} \int_{0}^{\infty} \frac{d u}{u^{3}} \frac{q B u}{\tan (q B u)} e^{-i \frac{s^{2}}{4 u}}\right]^{2}+\frac{(q B)^{2}}{16 \pi^{4} s^{2}}
\end{aligned}
$$

\footnotetext{
${ }^{1}$ Notice that $\dot{\mathscr{F}}_{11}(\omega)=\dot{\mathscr{F}}_{22}(\omega)$ for our choice of the field along the third axis.
} 
and $G_{11}^{+}(s)=G_{22}^{+}(s)$. These results are exact for free fermions in external magnetic field in the massless limit.

To compute the response function one needs to take into account $s \rightarrow s-i \varepsilon$ prescription corresponding to definition of the Wightman function (2.4) and switch on the temperature introducing sum over periodic shifts in imaginary time with $\beta=1 / k T$ and Fermi-Dirac statistics factor $(-1)^{k}$ for fermions (see, e.g. [13], [14]):

$$
\delta \dot{\mathscr{F}}(\omega)=\dot{\mathscr{F}}_{33}(\omega)-\dot{\mathscr{F}}_{11}(\omega)=-\frac{(q B)^{2}}{8 \pi^{4}} \int_{-\infty}^{+\infty} d s e^{-i \omega s}\left[\sum_{k=-\infty}^{+\infty} \frac{(-1)^{k}}{(s-i \varepsilon+i k \beta)}\right]^{2}
$$

Taking into account that $\sum_{k=-\infty}^{\infty} \frac{(-1)^{k}}{x+i k}=\frac{\pi}{\sinh \pi x}$ and doing the integral with the help of residues (see, e.g. [ㅁ] ]):

$$
\int_{-\infty}^{\infty} \frac{e^{-i \omega s} d s}{\sinh ^{2 n}(s-i \varepsilon)}=\frac{(-1)^{n}}{(2 n-1) !}\left(\frac{2 \pi}{\omega}\right) \frac{1}{e^{\pi \omega}-1} \prod_{l=1}^{n}\left(\omega^{2}+4(n-l)^{2}\right)
$$

one gets

$$
\delta \dot{F}(\omega)=\frac{(q B)^{2}}{4 \pi^{3}} \frac{\omega}{e^{\beta \omega}-1}
$$

Expression (2.14) is the main result of this section. It is positive, which corresponds to the fact that the detector measuring the current along magnetic field clicks more often than measuring perpendicular currents. It is also worth noticing the change of statistics from Fermi-Dirac to BoseEinstein - what is relevant is the statistic of operators whose fluctuations are being measured by the detector (Bose-currents in our case) and not the statistics of primary fluctuating fields.

The fact that current fluctuations are suppressed in perpendicular direction is obvious from general physics: the charged particle moving in the orthogonal plane is deflected by the magnetic field (or, using quantum mechanical language, confined to Landau levels). What is less obvious is that fluctuations along the field are enhanced (exactly by the same amount), since classically (i.e. neglecting spin effects) magnetic field has no influence on a charge moving in parallel direction. This enhancement is caused by spin interaction with the magnetic field and, to our view, can legally be called a particular case of CME.

It is instructive to compare (2.14) with fluctuation pattern without magnetic field. The latter can easily be obtained from (․ㅣ) or (‥T) putting $B=0$. The result reads:

$$
\dot{\mathscr{F}}^{(0)}(\omega)=\frac{1}{60 \pi^{3}} \frac{\omega}{e^{\beta \omega}-1}\left(\omega^{2}+4\left(\frac{2 \pi}{\beta}\right)^{2}\right)\left(\omega^{2}+\left(\frac{2 \pi}{\beta}\right)^{2}\right)
$$

The ratio is maximal for $\omega \rightarrow 0$ but even in this region it is rather small numerically:

$$
\frac{\delta \dot{\mathscr{F}}(0)}{\dot{\mathscr{F}}(0)(0)}=\frac{15}{64 \pi^{4}} \cdot \frac{(q B)^{2}}{T^{4}} \approx 0.0024 \cdot \frac{(q B)^{2}}{T^{4}}
$$

At large magnetic fields the fluctuations are frozen in $1-2$ plane, so that

$$
G_{11}^{+}(s) \rightarrow 0 ; G_{33}^{+}(s)=-\frac{(q B)^{2}}{8 \pi^{4} s^{2}}
$$


and correspondingly

$$
\frac{\dot{\mathscr{F}}_{33}(\omega)-\dot{\mathscr{F}}_{11}(\omega)}{\dot{\mathscr{F}}_{33}(\omega)+\dot{\mathscr{F}}_{11}(\omega)} \rightarrow 1
$$

The pattern (2.17), (2.18) is easy to understand in term of the corresponding energy-momentum tensor. Indeed, at zero magnetic field one has the standard thermal pressure for massless fermions

$$
T_{11}=T_{22}=T_{33}=\frac{7 \pi^{2} T^{4}}{180}
$$

which is isotropic. At large magnetic field, however, all the pressure is along the magnetic field and there is no pressure in orthogonal directions:

$$
T_{11}=T_{22} \rightarrow 0 ; T_{33}=\frac{q B T^{2}}{12}
$$

It is interesting to note that for strong but slowly varying magnetic field the plasma as a whole is to experience buoyancy force in the direction of the field gradient:

$$
f_{3}=-\int_{V} d^{3} x \frac{\partial T_{33}}{\partial z}
$$

This effect can be called magnetic Archimedes force. Since in real scattering events the fields are indeed highly inhomogeneous, this effect could be important for such phenomena as charge dependence of the elliptic flow etc. These questions are to be explored in future research.

\section{Parity violation and decoherence}

It is rather clear that if one is monitoring some $\mathbf{P}$-odd observable, it can lead to nonzero result for measurement of correlated $\mathbf{P}$-odd quantity. The simplest way to see it is to use a language of decoherence functionals ([ए8], see also [ए]]) and path integral formalism. Generally, for some filter function $\alpha[\Phi]$ the amplitude is given by

$$
\Psi[\alpha]=\int \mathscr{D} \Phi \alpha[\Phi] e^{i S[\Phi]}
$$

The most important missing ingredient in our discussion so far is the fact that in strong interaction domain the singlet axial vector current is not conserved because of triangle nonabelian anomaly:

$$
\partial^{v} j_{v}^{5}(x)=-\eta(x)=-\frac{g^{2} N_{f}}{16 \pi^{2}} G_{\alpha \beta}^{a}(x) \tilde{G}^{a \alpha \beta}(x)
$$

We are interested to find common distribution for the vector current and some $\mathbf{P}$-odd quantity, which we have chosen in this section to be the field $\eta(x)$ from (B.2). The corresponding amplitude reads:

$$
\Psi[\lambda, \kappa]=\int \mathscr{D} \bar{\psi} \mathscr{D} \psi \mathscr{D} A_{\mu} e^{i S_{Q C D}+i \int d x \lambda(x) n_{\mu} j^{\mu}(x)+i \int d x \kappa(x) \eta(x)}
$$

The vector current is given by the standard expression $j_{\mu}=\bar{\psi} Q \gamma_{\mu} \psi$, where $Q$ is quark electric charges diagonal matrix in flavor space. The closed-time-path functional is given by

$$
e^{i W\left[\lambda, \kappa ; \lambda^{\prime}, \kappa^{\prime}\right]}=\Psi[\lambda, \kappa] \Psi^{*}\left[\lambda^{\prime}, \kappa^{\prime}\right]
$$


and the mean current is

$$
\left\langle n_{\mu} j^{\mu}(x)\right\rangle[\lambda, \kappa]=-\left.i \frac{\delta}{\delta \lambda(x)} e^{i W\left[\lambda, \kappa ; \lambda^{\prime}, \kappa^{\prime}\right]}\right|_{\substack{\kappa=\kappa^{\prime} \\ \lambda=\lambda^{\prime}}}
$$

It is a functional of $\mathbf{P}$-even field $\lambda(x)$ and $\mathbf{P}$-odd field $\kappa(x)$.

It is easy to compute $\Psi[\lambda, \kappa]$ in Gaussian approximation and get expression for the induced current. As a model example we take concrete profile for the $\kappa$-field, corresponding to timedependent 3-dimensional "decoherence volume" $V\left(y_{0}\right)$ :

$$
\kappa(y)=\kappa \cdot f_{V}\left(y_{0}, \mathbf{y}\right)=\kappa \cdot \int_{V\left(y_{0}\right)} d^{3} \mathbf{w} \delta^{(3)}(\mathbf{y}-\mathbf{w})
$$

It leads to the following expression for the current parallel to magnetic field:

$$
\left\langle j_{3}(x)\right\rangle[0, \kappa]=\frac{N_{c} \operatorname{Tr} Q^{2}}{2 \pi^{2}} \cdot(\kappa B) \cdot \dot{f}_{V}\left(x_{0}, \mathbf{x}\right) \cdot e^{-\int d p \kappa(p) \mathfrak{I}\left\{\Pi^{5}(p)\right\} \kappa(-p)}
$$

where

$$
\Pi^{5}(p)=i \int d x e^{i p x}\langle T\{\eta(x) \eta(0)\}\rangle
$$

and we switched off the $\mathbf{P}$-even filter $(\lambda=0)$, but has kept the $\mathbf{P}$-odd one. The above expression is a generalization of $(\mathbb{L}, 2)$ with the field $\kappa($ (B. $)$ being direct analog of time-dependent $\theta$-term. The current is linear both in $\kappa$ and in $B$ and vanishes being integrated over $\kappa$ in symmetric limits. Notice that the current flows only inside the volume (where the measurement has been done) and what is important it has a maximum in $\kappa$. This maximum in $\kappa$ transforms into optimal (i.e. maximizing the current) speed of 3-volume expansion: for too slow expansion the current is small due to $\dot{f}_{V}\left(x_{0}, \mathbf{x}\right)$ factor, while for too fast one would expect strong damping from the region of effective large timelike momenta in decoherence exponent.

\section{Conclusion}

We discussed the phenomenon of CME using the ideas of quantum measurements theory. First, it was shown that in the simplest case of free massless fermions the nonzero asymmetry of electric current fluctuations in magnetic field is detected by the standard Unruh-DeWitt detector at rest. This asymmetry (2.14) is of desired sign (i.e. detector clicks more often measuring current components along the field than in perpendicular plane) but numerically it is rather weak for usual thermal fluctuations even in its maximum. We find it remarkable that this asymmetry is exactly quadratic in magnetic field $B$ and gets no higher order corrections. Despite the effect is not linear in $B$ as conventional (abelian) triangle anomaly is, its origin can be traced to the same asymmetry in the fermion Green's function in the magnetic field which is responsible for CME in the standard approach. Second, taking into account singlet axial vector current non-conservation due to nonabelian anomaly, we computed the electric current (B.7) along magnetic field under assumption that particular $\mathbf{P}$-odd quantity (dual field to the topological charge density in our example) can be treated as external (classical). This can be understood as a model for chiral chemical potential $\mu_{5}$ generation in quark-gluon dense and hot medium via decoherence along well known line of thought about classical features of intense gluon field in this system. 


\section{References}

[1] C. Vafa and E. Witten, "Restrictions on Symmetry Breaking in Vector-Like Gauge Theories," Nucl. Phys. B 234 (1984) 173.

[2] C. Vafa and E. Witten, "Parity Conservation in QCD," Phys. Rev. Lett. 53 (1984) 535.

[3] M. Dey, V. L. Eletsky and B. L. Ioffe, "Mixing of vector and axial mesons at finite temperature: an Indication towards chiral symmetry restoration,” Phys. Lett. B 252 (1990) 620.

[4] L. D. McLerran, E. Mottola and M. E. Shaposhnikov, "Sphalerons And Axion Dynamics In High Temperature Qcd," Phys. Rev. D 43 (1991) 2027.

[5] D. Kharzeev, R. D. Pisarski and M. H. G. Tytgat, "Possibility of spontaneous parity violation in hot QCD,” Phys. Rev. Lett. 81, 512 (1998) [arXiv:hep-ph/9804221].

[6] D. Kharzeev and R. D. Pisarski, "Pionic measures of parity and CP violation in high energy nuclear collisions,” Phys. Rev. D 61, 111901 (2000) [arXiv:hep-ph/9906401].

[7] D. Kharzeev, A. Krasnitz and R. Venugopalan, "Anomalous chirality fluctuations in the initial stage of heavy ion collisions and parity odd bubbles,” Phys. Lett. B 545, 298 (2002) [arXiv:hep-ph/0109253].

[8] D. Kharzeev, "Parity violation in hot QCD: Why it can happen, and how to look for it," Phys. Lett. B 633, 260 (2006) [arXiv:hep-ph/0406125].

[9] A. Vilenkin, “Equilibrium Parity Violating Current In A Magnetic Field,” Phys. Rev. D 22 (1980) 3080 .

[10] S. L. Adler, “Axial vector vertex in spinor electrodynamics,” Phys. Rev. 177 (1969) 2426.

[11] J. S. Bell and R. Jackiw, "A PCAC puzzle: pi0 -> gamma gamma in the sigma model," Nuovo Cim. A 60 (1969) 47.

[12] V. D. Orlovsky and V. I. Shevchenko, “Towards a quantum theory of chiral magnetic effect," Phys. Rev. D 82 (2010) 094032 [arXiv:1008.4977 [hep-ph]].

[13] V. Braginsky, F. Khalili, Quantum Measurement, Cambridge University Press, 1992.

[14] N. D. Birrell, P. C. W. Davies, Quantum fields in curved space, Cambridge University Press, 1984

[15] C. H. G. Bessa, J. G. Duenas and N. F. Svaiter, "Accelerated detectors in Dirac vacuum: the effects of horizon fluctuations," arXiv:1204.0022 [hep-th].

[16] J. S. Schwinger, “On gauge invariance and vacuum polarization,” Phys. Rev. 82 (1951) 664.

[17] T. Padmanabhan and T. P. Singh, "Response Of An Accelerated Detector Coupled To The Stress Energy Tensor,” Class. Quant. Grav. 4 (1987) 1397.

[18] R. P. Feynman and F. L. Vernon, Jr., "The Theory of a general quantum system interacting with a linear dissipative system," Annals Phys. 24 (1963) 118 [Annals Phys. 281 (2000) 547].

[19] E. Calzetta and B. L. Hu, "Correlations, decoherence, dissipation, and noise in quantum field theory," hep-th/9501040.

[20] H.-P. Breuer, F. Petruccione, The theory of open quantum systems, Oxford University Press, 2002 